\title{
PIODERMA GANGRENOSO GIGANTE DE CURSO FULMINANTE ASOCIADO A ENFERMEDAD INFLAMATORIA INTESTINAL*
}

\author{
Drs. Guillermo Bannura C. ${ }^{1}$, Alejandro Barrera E. ${ }^{1}$, Carlos Melo L. ${ }^{1}$ \\ ${ }^{1}$ Servicio y Departamento de Cirugía. Hospital Clínico San Borja Arriarán. Campus Centro, \\ Facultad de Medicina. Universidad de Chile. \\ Santiago, Chile.
}

\begin{abstract}
\section{Giant pyoderma gangrenosum associated with an inflammatory bowel disease}

Background: Pyoderma gangrenosum (PG) is an uncommon neutrophilic dermatosis that presents as an inflammatory and ulcerative disorder of the skin. Case report: We report a 23 years old male subjected to a total colectomy and ileostomy due to a toxic megacolon associated with an inflammatory bowel disease. Ten months later, he suffered an infected abdominal wall dehiscence after a surgical intervention for a bowel obstruction. The patient presented confluent ulcerated skin lesions surrounding the ileostomy and drainage sites, associated with severe malaise and without response to antimicrobial treatment. Presuming the presence of a giant and fulminant pyoderma gangrenosum associated with Crohn's disease, the patient was treated with adrenal steroids and immunosuppressive agents. One month later an enterocutaneous fistula appeared. Thereafter the patient experienced a slow but progressive recovery with a complete healing of the skin lesion and closure of enterocutaneous fistula after four years of immunosuppressive and anti-tumor necrosis factor treatment. The basis of the treatment of this patient was the use of immunosuppressive agents.
\end{abstract}

Key words: Pyoderma gangrenosum, inflammatory bowel disease, immunosuppression.

\section{Resumen}

Introducción: El pioderma gangrenoso (PG) es una enfermedad cutánea crónica, probablemente de etiología autoinmunitaria, que se manifiesta en la mayoría de los casos como una úlcera dolorosa. Caso clínico: Se presenta el caso de un paciente de 23 años sometido a una colectomía total e ileostomía por megacolon tóxico asociado a una enfermedad inflamatoria intestinal. A los 10 meses, tras una intervención por obstrucción intestinal presenta una infección con dehiscencia de la pared abdominal y grandes zonas ulceradas confluentes peri-ileostómicas y en el sitio del drenaje, en el contexto de un grave compromiso del estado general que no responde a múltiples esquemas antibióticos. Por la forma clínica de presentación, el diagnóstico de esta condición fue elusivo. Con la colaboración de un equipo multidisciplinario y el diagnóstico presuntivo de pioderma gangrenoso (PG) gigante de curso fulminante asociado a una enfermedad de Crohn, se inicia

\footnotetext{
Los autores no refieren conflictos de interés.

Correspondencia: Dr. Guillermo Bannura C. gbannura@gtdmail.com
}

*Recibido el 1 de julio de 2013 y aceptado para publicación el 11 de octubre de 2013. 
corticoterapia asociado a inmunosupresores, que cambia el curso ominoso de la enfermedad, logrando una recuperación lenta pero progresiva del estado general y una cicatrización completa de la pared abdominal luego de 4 años de tratamiento y el cierre de una fístula entero-cutánea que surge en la evolución de la ulceración central. Conclusiones: Los pilares del tratamiento en este inusual caso de PG fueron la corticoterapia asociada a inmunosupresores en la fase aguda de la enfermedad, seguida de un agente biológico inhibidor del factor de necrosis tumoral (infliximab) en la fase de mantención.

Palabras clave: Pioderma gangrenoso, enfermedad inflamatoria intestinal, infliximab.

\section{Introducción}

El pioderma gangrenoso (PG) es una enfermedad cutánea crónica, probablemente de etiología autoinmunitaria, que se manifiesta en la mayoría de los casos como una úlcera dolorosa ${ }^{1}$. En el $50 \%$ de los casos está asociada a una enfermedad sistémica, especialmente las enfermedades inflamatorias del intestino (EEII), artritis reumatoide y discrasias sanguíneas ${ }^{2}$. EL PG paraileostómico es una variante del PG clásico descrito inicialmente por O'Loughlin y Perry ${ }^{3}$ en 1978, y se caracteriza por múltiples pústulas estériles rodeadas de un halo eritematoso que rápidamente progresan hasta formar una gran úlcera peri-ostómica. Se estima que esta complicación afecta al $0,6 \%$ de las ostomías, aparecen en promedio a los 18 meses de la confección y la mayoría de los casos ocurren en pacientes con una EEII ${ }^{4}$. Se presenta un caso de un PG para-ileostómica gigante que comprometía toda la pared abdominal en un paciente portador de una EEII.

\section{Caso clínico}

Paciente de 23 años, homosexual con trastorno depresivo adaptativo. En abril de 2008 es hospitalizado por una crisis grave de colitis ulcerosa que, a pesar del tratamiento, progresa a megacolon tóxico, por lo que es sometido a una colectomía total con ileostomía. El estudio anatomo-patológico del colon concluye: EEII tipo colitis ulcerosa con actividad acentuada. Reintervenido a los 10 meses por obstrucción intestinal por bridas, reingresa a los 10 días por infección del sitio quirúrgico superficial, caracterizada por dehiscencia total de la herida operatoria y un lago de pus, con grave compromiso del estado general, deshidratado, febril, con taquicardia, anemia intensa, más un proceso infeccioso necrotizante del periné con múltiples trayectos fistulosos. Evoluciona en forma tórpida sin respuesta a múltiples esquemas antibióticos ensayados. TC de abdomen revela una evisceración contenida sin colecciones. Rx Tórax muestra una neumonía multifocal con baciloscopias $\mathrm{y}$ hemocultivos negativos. Aseo quirúrgico bajo anestesia revela una extensa evisceración contenida, abundante secreción purulenta y pérdida de la fijación de la ileostomía a la pared abdominal (Figura 1). Biopsia de piel revela un infiltrado inflamatorio inespecífico. Es sometido a curación avanzada con sistema VAC. Una junta médica multidisciplinaria diagnostica un Pioderma gangrenoso para-ileostómico gigante en el contexto de una enfermedad de Crohn, por lo que se inicia corticoterapia con 100 mg hidrocortisona ev cada $8 \mathrm{~h}$ más azatioprina 100 $\mathrm{mg}$ cada $8 \mathrm{~h}$ por sonda nasoyeyunal, luego de lo cual se aprecia una lenta mejoría de su estado general. El 10 de julio de 2009 es capaz de ingerir por vía oral volúmenes hasta $2.600 \mathrm{ml}$ de Ensure al $20 \%$ y recibe prednisona $20 \mathrm{mg}$ en dosis decrecientes.

Un mes después se constata la aparición de una fístula intestinal (Figura 2) de débito moderado (100-300 ml en $24 \mathrm{~h}$ ) en el tejido granulatorio de la herida principal y la ileostomía se aprecia más fija en la pared abdominal. Persiste con alzas térmicas de hasta $39,4^{\circ} \mathrm{C}$ con menor repercusión sistémica, aunque la PCR sigue elevada (103). Luego de la $4^{\mathrm{a}}$ dosis de Infliximab, presenta cuadro de compromiso de estado general, somnolencia, fiebre, ictericia (Bil total 3,5, directa 3,4 con FA y transaminasas normales) asociado a pancitopenia, por lo que se suspende la azatioprina y la mesalazina y se reinicia corticoides por vía ev. Tras 8 días de antibioterapia

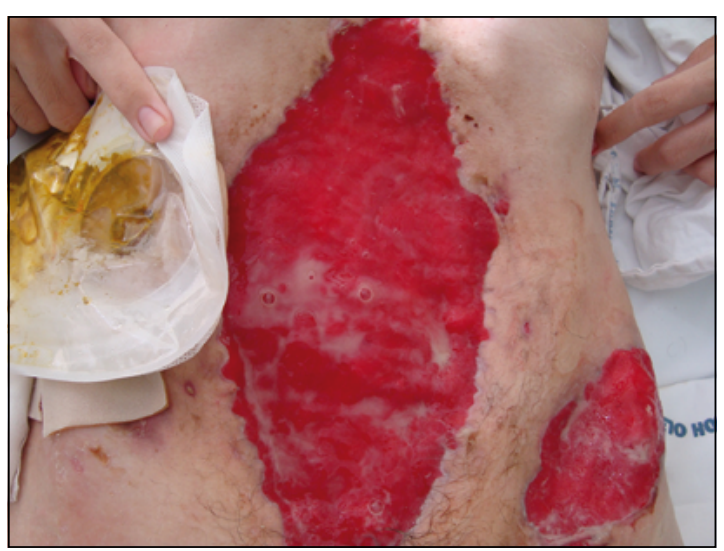

Figura 1. Lesiones ulceradas extensas peri-ileostómicas, incisión media supra-infraumbilical y sitio de drenaje. 


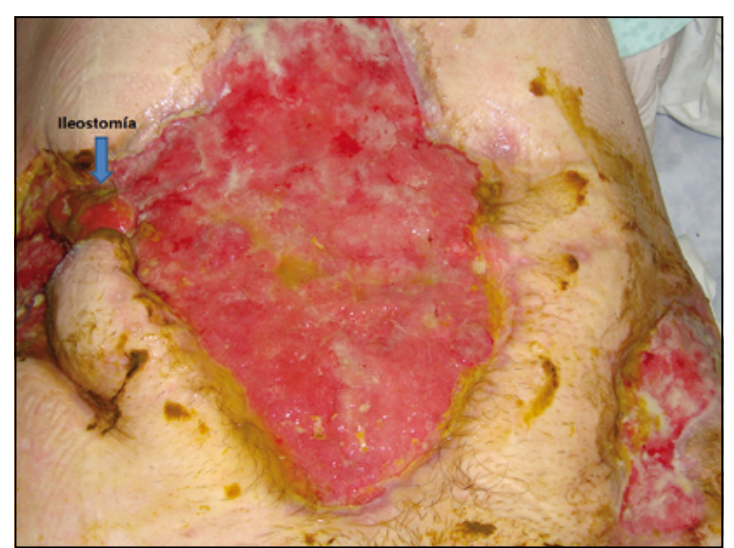

Figura 2. Úlcera peri-ileostómica y central confluentes. PG complicado con fístula enterocutánea

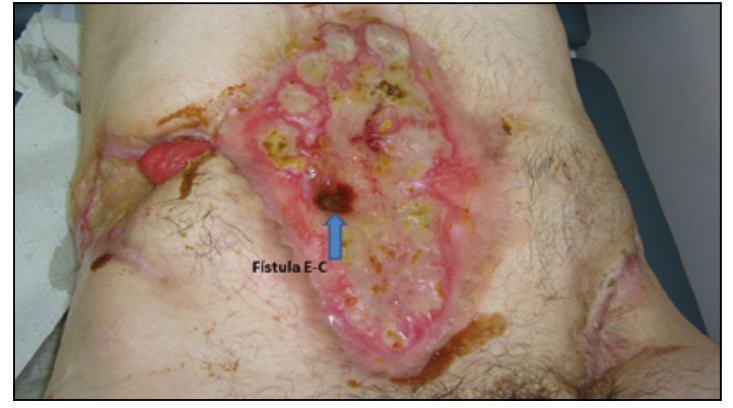

Figura 3. Epitelización lenta de las úlceras con puente cutáneo. Fístula activa.

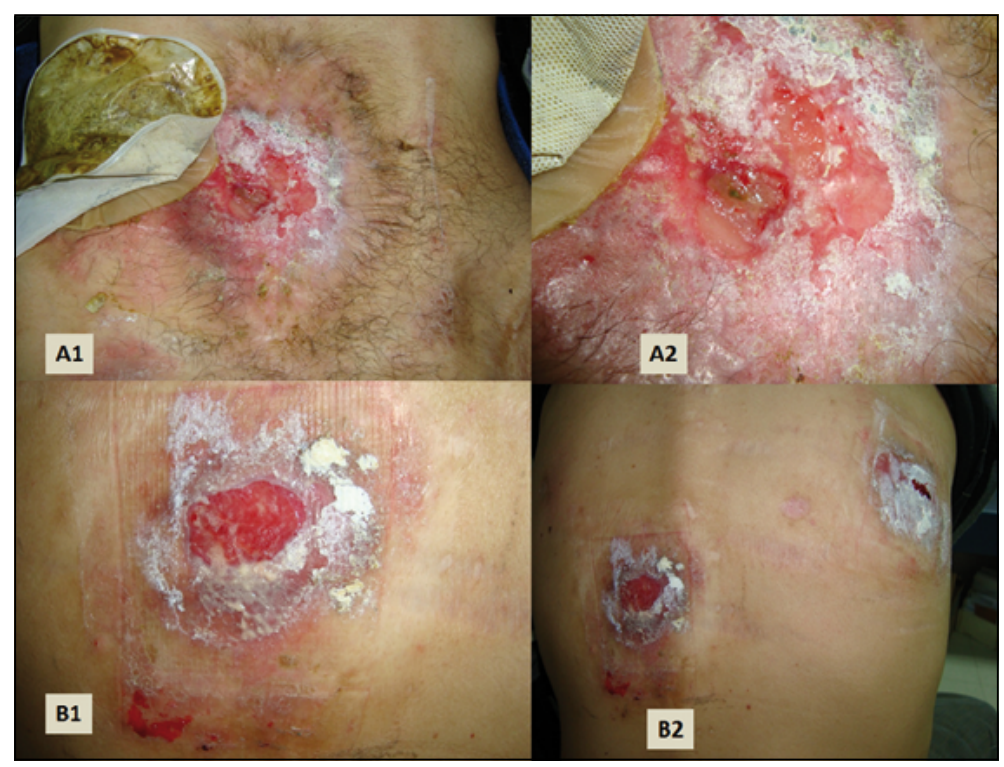

Figura 4. A1-A2. Cicatrización más avanzada con fístula activa de bajo débito. B1-B2. Aparición de úlceras dorsales compatible con PG. con amikacina, vancomicina e imipenem persiste con fiebre hasta $39^{\circ} \mathrm{C}$ y neutropenia febril sin foco claro. Días después mejora el recuento de blancos $\mathrm{y}$ el paciente tolera bien el régimen liviano, evoluciona afebril y recibe prednisona $60 \mathrm{mg}$ por día con una reducción franca de la zona cruenta con epitelización centrípeta (Figura 3). Alta en mejores condiciones generales y locales luego de 169 días de hospitalización.

Catorce meses después se constata una franca mejoría de las condiciones generales y nutritivas, signos de Cushing iatrogénico y una reducción significativa de las zonas cruentas (zona del drenaje izquierdo y área peri-ileostómicas epitelizadas), apreciándose una cicatrización casi completa con fístula activa con indicios de contenido intestinal, con la aparición de 2 lesiones ulceradas en el dorso que se interpretan como nuevas manifestaciones del PG (Figura 4). Completa 18 meses con Infliximab de mantención (cada 8 semanas) y azatioprina $150 \mathrm{mg}$ diarios. Dos meses después control revela lesiones del dorso cicatrizadas y fístula intestinal inactiva. (Figuras 5 y 6). Estudio del ano-recto desfuncionalizado muestra actividad inflamatoria con úlceras y múltiples pseudopólipos. El paciente rechaza la proctectomía. 


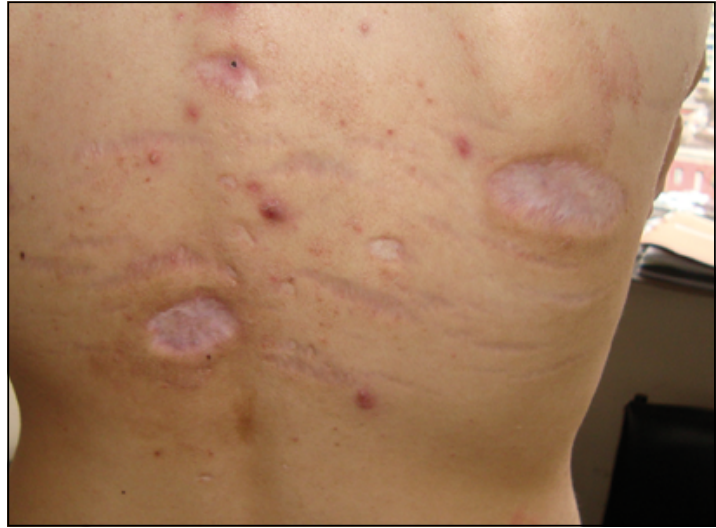

Figura 5. Cicatrización completa de úlceras dorsales.

\section{Discusión}

El PG clásicamente presenta 4 formas clínicas principales: ulcerosa (la más común), pustulosa, ampollosa y vegetante, a las cuales se agrega el PG periostómico, variedades que afecta menos del $1 \%$ de las ostomías; habitualmente son de curso autolimitado y sólo requieren de un manejo local ${ }^{5}$. El caso que se presenta tuvo un curso fulminante y en una vasta extensión que comprometió toda la pared abdominal, con desprendimiento de la ileostomía que debió ser fijada quirúrgicamente a la pared abdominal. El trauma repetido y la agresión quirúrgica se consideran como factores capaces de gatillar una respuesta inflamatoria desproporcionada, fenómeno conocido como patergia ${ }^{1-3}$. Las dimensiones y el grave compromiso del estado general con riesgo vital en este paciente determinó que el diagnóstico fuera tardío y, por tanto, hubo demora en iniciar el tratamiento adecuado.

La biopsia de la úlcera principal y luego de las lesiones del dorso fueron inespecíficas, lo que se considera lo habitual ${ }^{6}$. Hasta ahora, el diagnóstico del PG es fundamentalmente clínico y la biopsia de piel sirve en ocasiones para hacer el diagnóstico diferencial más que para certificar un PG.

EL PG perilieostómico se presenta principalmente en pacientes portadores de una EEII, especialmente colitis ulcerosa ${ }^{7}$. Aunque la biopsia del colon resecado en este paciente fue informada como colitis ulcerosa, la evolución de la enfermedad y el grave compromiso de la región anorrectal y perineal sugieren una enfermedad de Crohn.

El tratamiento del PG paraileostómico depende de la magnitud de las úlceras y el compromiso sistémico. Se inicia con manejo local con protectores de la piel que faciliten la adherencia de las prótesis

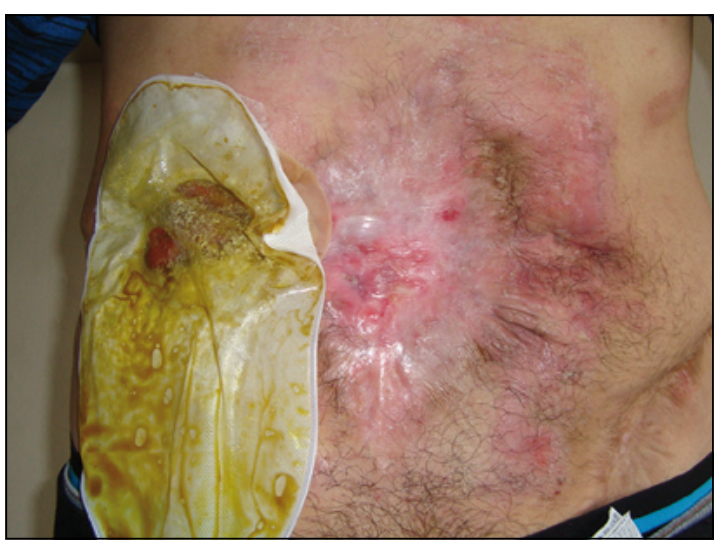

Figura 6. Cicatrización completa de la pared abdominal. Fístula entero-cutánea cerrada.

a la piel afectada, debiendo agregar corticoides sistémicos e inmunosupresores en casos más graves para lograr el control de la enfermedad ${ }^{8}$. En la fase de mantención destacan los antagonistas del factor de necrosis tumoral, que a pesar de los costos están indicados en casos refractarios al tratamiento habitual, además de ser efectivos contra la enfermedad de base9. Sin embargo, se han descrito casos de PG periileostómico que se presentan durante el tratamiento con infliximab ${ }^{10}$.

\section{Agradecimientos}

Al Dr. Carlos Quintana Villar, Pontificia Universidad Católica de Chile.

\section{Referencias}

1. Ruocco E, Sangiuliano S, Gravina AG, Miranda A, Nicoletti G. Pyoderma gangrenosum: an updated review. J Eur Acad Dermatol Venereol. 2009;23:1008-17.

2. Callen JP, Jackson JM. Pyoderma gangrenosum: an update. Rheum Dis Clin North Am. 2007;33:787-802.

3. O'Loughlin S, Perry HO. A diffuse pustular eruption associated with ulcerative colitis. Arch Dermatol. 1978;114:1061-4.

4. Lyon CC, Smith AJ, Beck MH, Wong GA, Griffiths CE. Parastomal pyoderma gangrenosum: clinical features and management. J Am Acad Dermatol. 2000;42:9921002.

5. Hevia H, Suárez J, Vergara MT. Pioderma gangrenoso periileostómico: caso clínico. Rev Med Chile 2004;132:747-9.

6. Fernández-Pulido C, García Patos-Briones V. Pioderma gangrenoso. Diagnóstico y tratamiento. Piel 
2008;23:24-9.

7. Poritz LS, Lebo MA,k Bobb AD, Ardell CM, Koltun WA. Management of periostomal pyoderma gangrenosum. J Am Coll Surg. 2008;206:311-5.

8. Reichrath J, Bens G, Bonowitz A, Tilgen W. Treatment recommendations for pyoderma gangrenosum: an evidence-based review of the literature based on more than 350 patients. J Acad Dermatol. 2005;53:273-83.
9. Zold E, Nagy A, Devenyi K, Zeher M, Barta Z. Successful use of adalimumab for treating fistulizing Crohn's disease with pyoderma gangrenosum: two birds with one stone. World J Gastroenterol. 2009;15:2293-5.

10. Jaimes-López N, Molina V, Arroyave JE, Vásquez LA, Ruiz AC, Castano R, et al. Development of pyoderma gangrenosum during therapy with infliximab. J Dermatol Case Report 2009;3:20-3. 